Polish Journal of Microbiology

2011, Vol. 60, No 2, 155-161

ORGINAL PAPER

\title{
Species-Specific Sensitivity of Coagulase-Negative Staphylococci to Single Antibiotics and Their Combinations
}

\author{
GRAŻYNA SZYMAŃSKA ${ }^{1 *}$, MAGDALENA SZEMRAJ ${ }^{2}$ and ELIGIA M. SZEWCZYK \\ ${ }^{1}$ Department of Biosynthesis of Drugs, ${ }^{2}$ Department of Pharmaceutical Microbiology \\ Medical University of Łódź, Poland
}

Received 25 September 2010, revised 2 April 2011, accepted 4 April 2011

\begin{abstract}
The activity of $\beta$-lactam antibiotics (oxacillin, cloxacillin, cephalotin), vancomycin, gentamicin and rifampicin applied in vitro individually and in combination against 37 nosocomial methicillin-resistant strains of coagulase-negative staphylococci (CNS) was assessed to demonstrate the heterogeneity of this group of bacteria and estimate the chance of the efficacy of such therapy. The strains belonged to four species: Staphylococcus epidermidis, Staphylococcus haemolyticus, Staphylococcus cohnii, Staphylococcus hominis. They originated from a hospital environment and from the skin of medical staff of the intensive care unit of a paediatric ward at a university hospital. All strains were methicillin-resistant, according to CLSI standards, but individual strains differed in $\mathrm{MIC}_{\mathrm{OX}}$ values. Susceptibility to other tested antibiotics was also characteristic for the species. The increased susceptibility to antibiotics in combinations, tested by calculating the fractional inhibitory concentration (FIC) index, concerned 26 out of 37 investigated strains and it was a feature of a particular species. Combinations of vancomycin and cephalotin against S. epidermidis and oxacillin with vancomycin were significant, as well as cephalotin and rifampicin in growth inhibition of multiresistant S. haemolyticus strains.
\end{abstract}

Ke y w ords: Staphylococcus sp., antibiotic synergism, methicillin-resistant CNS, staphylococci infections treatment

\section{Introduction}

Coagulase-negative staphylococci (CNS) have been regarded as a threat since the 80 s of the last century due to advances in medicine and implementation of new medical methods. This type of infection is difficult to diagnose as it is often not easy to distinguish an etiological disease factor from a natural flora contamination. Recently, much more attention has been paid to the need of CNS species identification and searching for a connection between species affiliation and the clinical importance of particular staphylococci (Tan et al., 2006; Hamels et al., 2001).

CNS are the leading causes of nosocomial bloodstream infections (Chandran and Rennie, 2005; Frigatto et al., 2005). According to Finkelstein et al., (2002) morbidity among patients with CNS bacteriemia amounts to $16 \%$, hence investigations concerning differences in resistance profiles of particular species of this group, especially multi-resistant strains are of particular significance. The increase of bacterial resistance forces rational antibiotic therapy in order to maintain the efficacy of these drugs for as long as possible (Wang and Lipstich, 2006). Methods of alternative therapy against these infections are being sought. One of them can be combination therapy (Dawis et al., 2003; Guerrero and Gorgolas, 2006; Miranda-Nowales et al., 2006).

In the presented investigations, the activity of $\beta$-lactam antibiotics (oxacillin, cloxacillin, cephalotin), vancomycin, gentamicin and rifampicin applied in vitro as single or in combination against nosocomial methicillin-resistant CNS belonging to S. epidermidis, S. haemolyticus, S. cohnii and S. hominis was investigated.

\section{Experimental}

Materials and Methods

37 methicillin-resistant CNS from our own collection were tested. They belonged to S.epidermidis $(\mathrm{n}=12)$, S. haemolyticus $(\mathrm{n}=9)$, S. cohnii $(\mathrm{n}=10)$, $S$. hominis $(n=6)$. The strains originated from hospital environment and from the skin of medical staff of the intensive care unit at a paediatric ward of a university hospital. Identification to the species level was performed with API-Staph System (BioMérieux). Only a single isolate per patient was tested. Methicillin resistance was detected phenotypically with a cefoxitin test $\left(\mathrm{FOX}_{30}-\right.$ Becton-Dickinson) and confirmed by mecA

\footnotetext{
* Corresponding author: G. Szymańska, phone: +48 4267793 01; fax: +48 4267793 00; e-mail: grazyna.szymanska@umed.lodz.pl
} 
gene detection in PCR method (PK14 kit - DNAGdańskII) (Van Griethuysen et al., 1999). $\beta$-lactamase was detected in a cefinase test (Becton-Dickinson). Strains were stored frozen in glycerol-broth at the temperature of $-70^{\circ} \mathrm{C}$.

Strain susceptibility tests were performed according to the standards of the CLSI (2008) by the MIC microdilution tests using oxacillin (OX), cloxacillin (CX), cephalotin (CF), gentamicin (GM), vancomycin (VA) and rifampicin (RA) (Fluka and Sigma) in MuellerHinton broth (BioMérieux) without $2 \% \mathrm{NaCl}$. MIC is the lowest concentration of antibiotic that yielded no visible growth after incubation at $35^{\circ} \mathrm{C}$ for $24 \mathrm{~h}$.

Activity of antibiotics in combination was checked, at first using disc sensitivity tests, where discs (all from Becton-Dickinson) were placed on Mueller-Hinton agar plates at a distance of $19-20 \mathrm{~mm}$ in pairs: $\mathrm{OX}_{1}+\mathrm{VA}_{30}$, $\mathrm{CF}_{30}+\mathrm{VA}_{30}, \mathrm{CF}_{30}+\mathrm{RA}_{5}, \mathrm{CF}_{30}+\mathrm{GM}_{10}$ and $\mathrm{CF}_{30}+\mathrm{CX}_{1}$. Subsequently, strains forming characteristic elongated shape of inhibit growth zones around discs with antibiotics were tested in a microdilution synergy test. Combinations of antibiotics at appropriate concentrations in Mueller-Hinton broth were tested in 96-well microtiter plates (Kartell). Plates were incubated at $35^{\circ} \mathrm{C}$ for $24 \mathrm{~h}$ and Fractional Inhibitory Concentration (FIC) index was calculated. FIC is MIC of drug in combination divided by the MIC of drug acting alone (MIC of drug A in combination with drug B/MIC of drug $\mathrm{A}$ alone). The sum of the FICs of both antibiotics gave the FIC index. Synergism was identified when the FIC index was $\leq 0.5$ (Climo et al., 1999; Dawis et al., 2003). All tests were performed at least twice. Reference strains were S. aureus ATCC 29213 and ATCC 25923. Quantitative variables were calculated using the Kruskal-Wallis test.

\section{Results}

All investigated strains were methicillin-resistant according to CLSI standards but individual strains presented different levels of oxacillin susceptibility, expressed in $\mathrm{MIC}_{\mathrm{OX}}$ values (Table I). The greatest number of strains with low MIC $(\leq 4 \mathrm{mg} / \mathrm{l})$ was found among S. hominis, with an average MIC (8-128 mg/l) among S. epiderminis and with high MIC $(\geq 256 \mathrm{mg} / \mathrm{l})$ among S. cohnii. There were significant differences in MIC values (from 0.5 to $2048 \mathrm{mg} / \mathrm{l}$ ) within species, particularly in S. haemolyticus. Nevertheless, the differences in antibiotic-resistance between species were clearly visible and statistically important in the Kruskal-Wallis test.

Ranges of MIC values for the tested antibiotics: cloxacillin, cephalotin, gentamicin, vancomycin and rifampicin were also different for individual species (Fig. 1). Resistant strains with high MIC values were the most numerous among S. haemolyticus. In spite of great standard deviations, it can be stated that statistically significant differences among species in their sensitivity to particular antibiotics not only concerned differences in absolute MIC values, but also sensitivity or resistance in the clinical sense, thus placing above or below the breakpoint values for individual antibiotics.

Using UPGMA (unweighted pair group mathematical averages), a dendrogram was created. The dendrogram grouped all investigated strains in relation to their antibiotic-susceptibility and the level of their resistance was expressed in MIC values. Strains formed several clusters and particular species were located in separated areas on the axis. S. haemolyticus strains were located mostly on the left side, S. epidermidis on the right, whereas S. cohnii and S. hominis in the central part of the dendrogram (Fig. 2).

In further experiments, the activity of antibiotics in combination was tested. Disc-diffusion method allowed to observe the occurrence of synergism between cephalotin and vancomycin (CF+VA), cephalotin and rifampicin $(\mathrm{CF}+\mathrm{RA})$, oxacillin and vancomycin $(\mathrm{OX}+\mathrm{VA})$, cephalotin and cloxacillin $(\mathrm{CF}+\mathrm{CX})$. In none of the investigated combinations either antagonism or addition occurred. For the strains in which, on the basis of growth inhibition zone shape around discs, the beneficial activity of antibiotics used in combination was observed, FIC index was calculated confirming or excluding synergism. Synergistic action was confirmed in most cases. It concerned 26 out of 37 investigated strains (Fig. 3). Most susceptible strains were among S. epidermidis - 10 out of 12 of the tested ones. The

Table I

MIC values of investigated species to oxacillin

\begin{tabular}{|l|c|c|c|c|}
\hline \multirow{2}{*}{$\begin{array}{c}\text { Species (number } \\
\text { of strains examined) }\end{array}$} & \multirow{2}{*}{$\begin{array}{c}\mathrm{MIC}_{\text {ox }}[\mathrm{mg} / \mathrm{l}] \\
\text { range in species }\end{array}$} & \multicolumn{3}{|c|}{ Number of strains of low, medium and high $\mathrm{MIC}_{\mathrm{ox}}$ values } \\
\cline { 3 - 5 } & $\leq 4 \mathrm{mg} / \mathrm{l}$ & $8-128 \mathrm{mg} / \mathrm{l}$ & $\geq 256 \mathrm{mg} / \mathrm{l}$ \\
\hline S. epidermidis (12) & $1-256$ & $5(42 \%)$ & $6(50 \%)$ & $1(8 \%)$ \\
\hline S. haemolyticus $(9)$ & $0.5-2048$ & $4(45 \%)$ & $2(22 \%)$ & $3(33 \%)$ \\
\hline S. cohnii (10) & $4-512$ & $1(10 \%)$ & $3(30 \%)$ & $6(60 \%)$ \\
\hline S. hominis $(6)$ & $0.5-64$ & $5(83 \%)$ & $1(17 \%)$ & 0 \\
\hline
\end{tabular}


Variable: Oxacillin

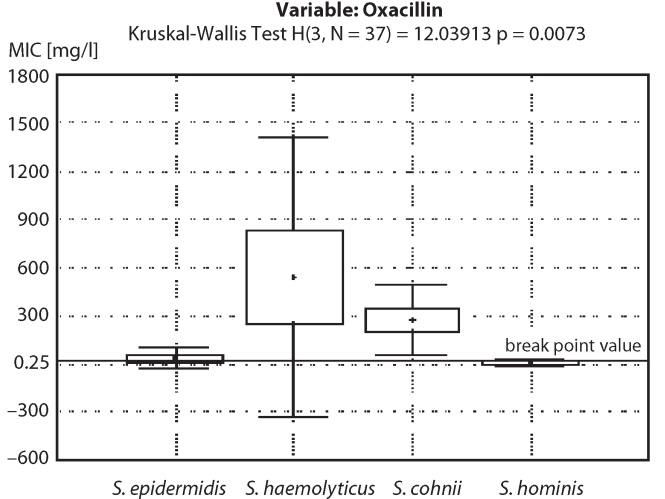

Variable: Cloxacillin

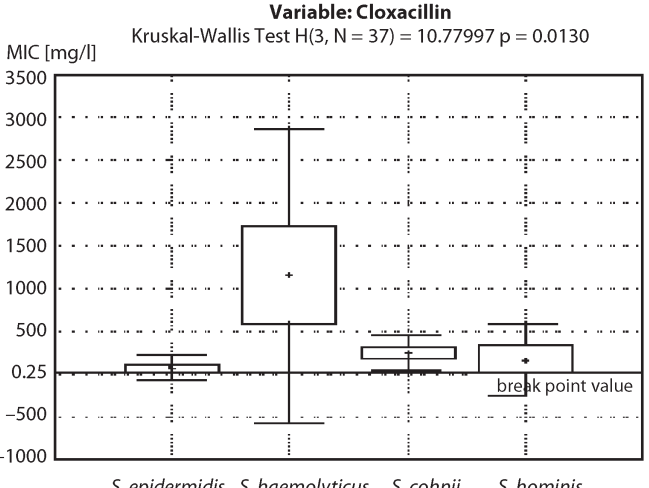

Variable: Cephalotin

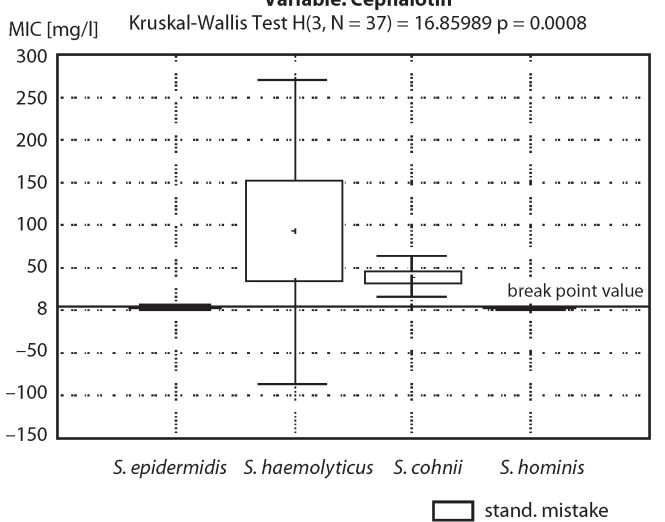

Variable: Gentamicin Kruskal-Wallis Test $\mathrm{H}(3, \mathrm{~N}=37)=16.141143 \mathrm{p}=0.0011$

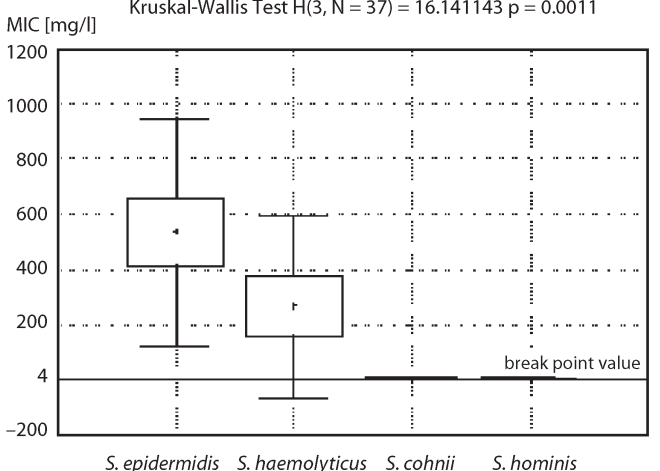

Variable: Vancomycin
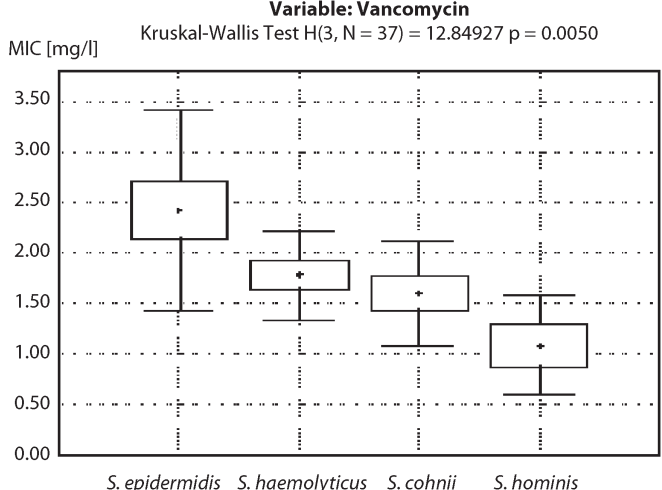

Variable: Rifampicin

MIC [mg/l] Kruskal-Wallis Test $\mathrm{H}(3, \mathrm{~N}=37)=27.37793 \mathrm{p}=0.0000$

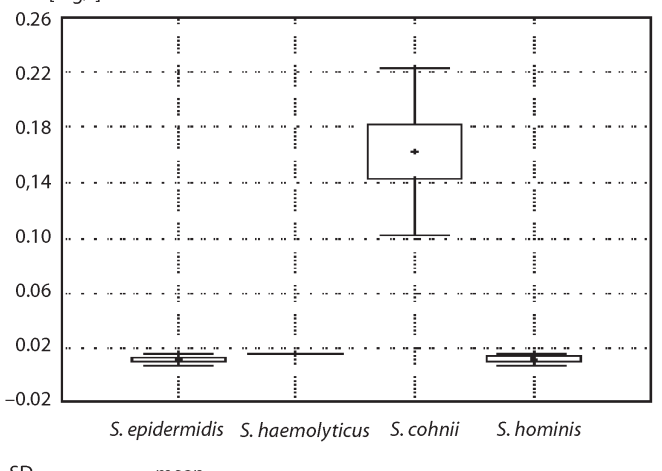

Fig. 1. Differences in MIC values of the tested antibiotics for strains of the investigated species. In the case of vancomycin and rifampicin, breakpoints were higher than the maximum points on a chart scale.

majority of them reacted this way to two or three pairs of antibiotics. Mostly, it was the combination of CF+VA. The same effect was achieved for most of the strains by $\mathrm{CF}+\mathrm{RA}$ binding. Only for S. epidermidis $\mathrm{CF}+\mathrm{CX}$ combination synergism occurred. However, for none of the S.epidermidis strains, synergism of $\beta$-lactams and gentamicin was determined. Synergistic activity of antibiotics against S. haemolyticus was observed for six out of nine tested strains. Mostly, combination of OX+VA (5 strains) presented synergism, less frequently combination of CF+RA (3 strains). Synergistic activity in eight out of ten of the tested S. cohnii strains presented CF+RA and in half of the tested strains a combination of $\mathrm{CF}+\mathrm{GM}$ occurred. In S. hominis synergism was noticed only against two strains and only for one pair of antibiotics (CF+ GM). Sensitivity to particular pairs of antibiotics was a feature connected with species. The combination presenting a synergistic effect in relation to the greatest number of tested strains was $\mathrm{CF}+\mathrm{RA}$. This combination was effective when MIC of 


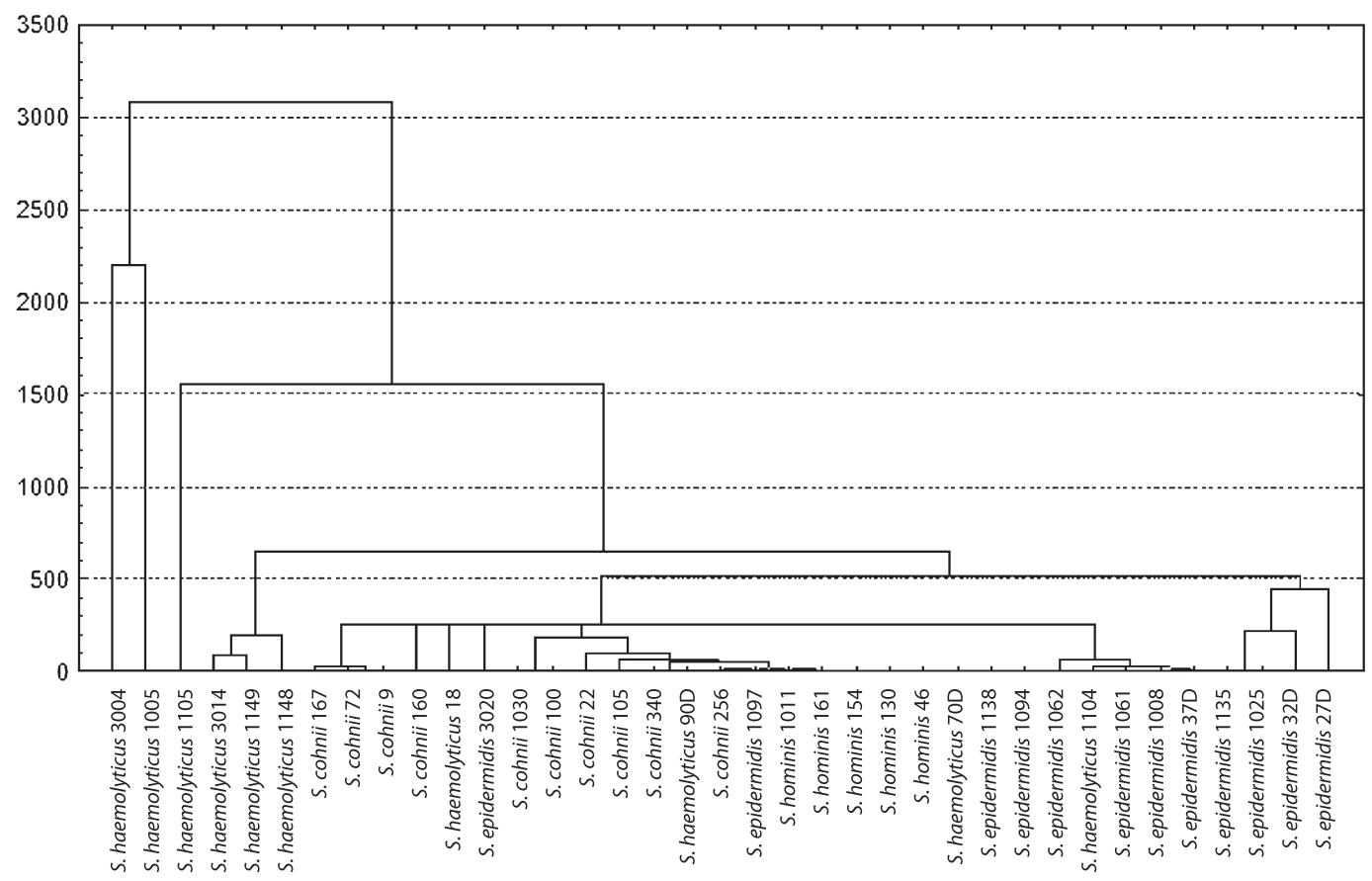

Fig. 2. Dendrogram presenting similarity in susceptibility to 6 antibiotics of 37 strains belonging to four tested species.
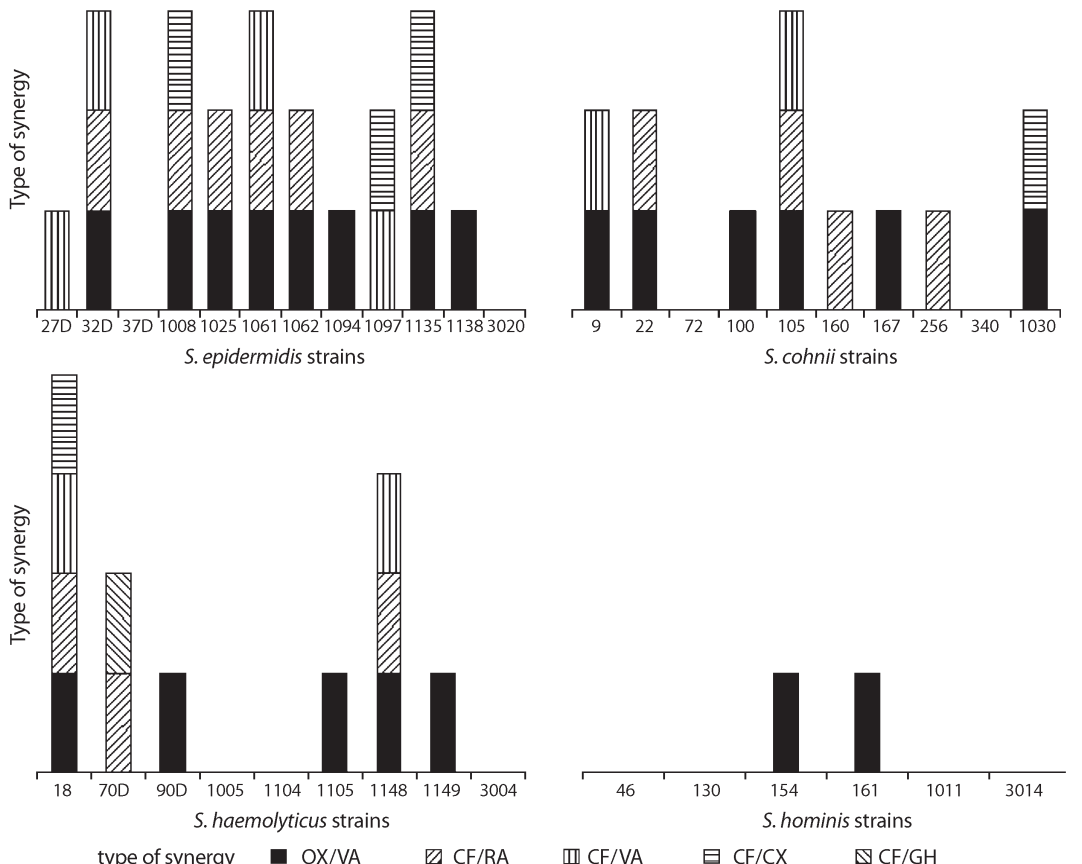

Fig. 3. Synergistic effect of antibiotics in combination against strains of the investigated CNS

cephalotin as a single antibiotic was $\leq 8 \mu \mathrm{g} / \mathrm{ml}$ (sensitive strain) but also when it was $\geq 32, \mu \mathrm{g} / \mathrm{ml}$ (resistant strain). Production of $\beta$-lactamases was a feature characteristic for almost all strains of the three tested species. The exception was S. cohnii strains, the majority of which did not produce $\beta$-lactamases. There was no connection between synergism occurrence and production of those enzymes.

Strains presenting a synergistic reaction to the highest number of antibiotics combinations are shown in 
Table II

Sensitivity of strains susceptible to a number of antibiotic combinations

\begin{tabular}{|c|c|c|c|c|c|c|c|c|}
\hline \multirow{2}{*}{ Strain } & \multicolumn{6}{|c|}{ MIC [mg/l] } & \multirow{3}{*}{$\begin{array}{l}\beta \text {-lactamase } \\
\text { production }\end{array}$} & \multirow{3}{*}{$\begin{array}{l}\text { Antibiotics in syner- } \\
\text { gistic combinations } \\
\text { MIC }[\mathrm{mg} / \mathrm{l}]\end{array}$} \\
\hline & OX & $\mathrm{CF}$ & $\mathrm{CX}$ & VA & RA & GM & & \\
\hline CLSI breakpoints & 0.25 & 8 & 0.25 & 4 & 1 & 4 & & \\
\hline \multirow[t]{3}{*}{ S. epidermidis 32D } & 256 & 8 & 128 & 2 & 0.008 & 1024 & & $\mathrm{OX}+\mathrm{VA}(16+0.25)$ \\
\hline & $\mathrm{R}$ & S & $\mathrm{R}$ & S & S & $\mathrm{R}$ & + & $\mathrm{CF}+\mathrm{VA}(2+0.125)$ \\
\hline & & & & & & & & $\mathrm{CF}+\mathrm{RA}(1+0.0019)$ \\
\hline \multirow[t]{3}{*}{ S. epidermidis 1008} & 8 & 8 & 32 & 4 & 0.008 & 512 & & $\mathrm{CF}+\mathrm{VA}(2+0.25)$ \\
\hline & $\mathrm{R}$ & S & $\mathrm{R}$ & S & S & $\mathrm{R}$ & - & $\mathrm{CF}+\mathrm{CX}(1+1)$ \\
\hline & & & & & & & & $\mathrm{CF}+\mathrm{RA}(2+0.0005)$ \\
\hline \multirow[t]{3}{*}{ S. epidermidis 1061} & 16 & 8 & 32 & 2 & 0.008 & 512 & & $\mathrm{OX}+\mathrm{VA}(4+0.25)$ \\
\hline & $\mathrm{R}$ & S & $\mathrm{R}$ & S & S & $\mathrm{R}$ & + & $\mathrm{CF}+\mathrm{VA}(2+0.0625)$ \\
\hline & & & & & & & & $\mathrm{CF}+\mathrm{RA}(2+0.0019)$ \\
\hline \multirow[t]{3}{*}{ S. epidermidis 1135} & 64 & 2 & 32 & 2 & 0.016 & 1024 & & $\mathrm{CF}+\mathrm{VA}(0.25+0.25)$ \\
\hline & $\mathrm{R}$ & S & $\mathrm{R}$ & S & S & $\mathrm{R}$ & + & $\mathrm{CF}+\mathrm{CX}(0.25+8)$ \\
\hline & & & & & & & & $\mathrm{CF}+\mathrm{RA}(0.5+0.00312)$ \\
\hline \multirow[t]{4}{*}{ S. haemolyticus 18} & 512 & 1 & 8 & 2 & 0.016 & 0.25 & & $\mathrm{OX}+\mathrm{VA}(128+0.5)$ \\
\hline & $\mathrm{R}$ & S & $\mathrm{R}$ & S & S & S & + & $\mathrm{CF}+\mathrm{VA}(0.125+0.0625)$ \\
\hline & & & & & & & & $\mathrm{CF}+\mathrm{CX}(0.125+0.125)$ \\
\hline & & & & & & & & $\mathrm{CF}+\mathrm{RA}(0.25+0.0019)$ \\
\hline \multirow[t]{3}{*}{ S. haemolyticus 1148} & 128 & 32 & 1024 & 2 & 0.016 & 256 & & $\mathrm{OX}+\mathrm{VA}(4+0.5)$ \\
\hline & $\mathrm{R}$ & $\mathrm{R}$ & $\mathrm{R}$ & S & S & $\mathrm{R}$ & + & $\mathrm{CF}+\mathrm{VA}(2+0.5)$ \\
\hline & & & & & & & & CF+RA $(8+0.00375)$ \\
\hline \multirow[t]{3}{*}{ S. cohnii 105} & 64 & 32 & 64 & 2 & 0.125 & 1 & & $\mathrm{OX}+\mathrm{VA}(16+0.5)$ \\
\hline & $\mathrm{R}$ & $\mathrm{R}$ & $\mathrm{R}$ & S & S & S & - & CF+GM (4+0.125) \\
\hline & & & & & & & & $\mathrm{CF}+\mathrm{RA}(4+0.0312)$ \\
\hline
\end{tabular}

$\mathrm{R}$ - resistant, $\mathrm{S}$ - sensitive

Table II, in which MIC values for single antibiotics, strain sensitivity in clinical sense and antibiotic combinations giving synergistic effect and MIC values achieved for antibiotics in pairs are presented.

The use of combinations of antibiotics gave an effective growth inhibition of the tested bacteria with simultaneously lowered antibiotic concentration. For S. epidermidis strains vancomycin concentration could be lowered 8-64 fold and for S. haemolyticus and S. cohnii 4-fold. Cephalotin could be applied mostly in the dose of 4-16 times lower than when it was applied individually.

\section{Discussion}

CNS include several dozens of species and are a heterogenic group of microorganisms, but only some of them are frequently isolated from humans. There is no doubt nowadays that these bacteria, especially methicillin-resistant strains, are important nosocomial pathogens. In this research strains belonging to four of these species were investigated. Three of those are species considered the most important in hospital infections
(S. epidermidis, S. haemolyticus, S. hominis), relatively often isolated from clinical samples (Kloos and Bannerman, 1994; Weinstein et al., 1998) and numerously represented in the hospital environment. S. cohnii strains are less frequent in clinical materials, but often occur in the hospital environment and on the skin of medical personnel. S. hominis may be considered a resistance reservoir in the environment (Kloos, 1997; Szewczyk et al., 2004). All strains were multiresistant and it could be assumed that this resistance was a result of selective pressure. Potentially, each of them could become the cause of a hospital infection.

The need to identify CNS species, wrongly treated as the same, is often perceived. It is stressed by many authors that such approach is a significant obstacle in research and diagnostics (Tacconelli et al., 2003). Nevertheless, there are still papers, even quite recent ones, in which all isolates from this group are treated equally (Kuti et al., 2008). There were also some radical opinions questioning antibiotic resistance evaluation of microorganisms from this group (Chandran and Rennie, 2005). This work was to defy such approach by showing the results of our research. 
Methicillin resistance estimation of CNS still rises doubts and discussion. This stems from the differences among particular species. The differences between novobiocin-sensitive and novobiocin-resistant species are clearly visible, however, the similarity of S. haemolyticus to the latter group is noticeable (Nowak et al., 2006; Frigatto et al., 2005). This is presumably connected with the content of different cassettes including mecA gene in staphylococci (Martins and Maria de Lourdes Cunha, 2007) and perhaps also with other features of these bacteria. There is an urgent need for research in this matter.

The multiresistant strains tested herein clearly show the differences in antibiotic susceptibility profiles of the tested species. It may be suspected that they also differ in genetic equipment. The results showed the noticeable resistance of strains of S. haemolyticus and S. cohnii. John et al., 2002, who conducted a research on the spectrum of 658 clinical staphylococcal isolates belonging to 13 species, pointed out that the highest levels of participation of isolates resistant to oxacyllin, erythromycin, klindamycin and telithromycin were presented by S. haemolyticus and S. epidermidis while for S. cohnii to quinupristin/dalfopristin. The presence of multiple plasmids in the cells was shown in the latter species (Szewczyk et al., 2004).

Sensitivity to cephalosporins maintained in methicillin-resistant staphylococci strains (S.epidermidis, S. hominis) was observed previously. In the research of Krediet et al., (1999) this feature was characteristic for 25 (almost 70\%) of the tested strains including all from S. epidermidis and S. hominis, five strains of S. haemolyticus and two of S. cohnii. The use of this antibiotic in CNS infection therapy should be analysed thoroughly, particularly because of earlier suggestions to apply it. All environmental strains of staphylococci tested by Szewczyk et al., (2000) were susceptible to cefuroxime and cefotaxime. $87 \%$ and $71 \%$ isolates from infants were respectively susceptible to two of these cephalosporins. All investigated strains of S. epidermidis from both groups were susceptible to cephalotin.

All of the strains from the collection tested in this research were sensitive to vancomycin and rifampicin, though clear differences in MIC values among species were visible (Fig. 1). Vancomycin is still frequently used in treatment of serious infections caused by gram-positive microorganisms, but stepping away from vancomycin therapy and replacing it with, e.g. cloxacillin both in cases of infections caused by S. aureus and CNS is recommended (Lawrence et al., 2005). As can be seen in the results presented in this work, therapy with cloxacillin would not meet the expectations in case of all species investigated CNS except $S$. haemolyticus, especially in combination with cephalotin. Lowered sensitivity to glycopeptides of S. aureus and S. epidermidis strains was described by authors who analysed CNS clinical isolates' sensitivity to vancomycin and claimed appearance of tolerance to that antibiotic and low sensitivity level (Walsh et al., 2001; Bourgeois et al., 2007).

Several studies have previously reported the synergistic effect of antibiotics on methicillin-resistant strains of staphylococci. Such studies have been undertaken mostly towards infections caused by multiresistant clinical strains i.e. MRSA (Dawis etal., 2003). Also research on treatment of infections with those bacteria in experimental models has been carried out. Fox et al. (2006), showed that using nafcillin and vancomycin in combination cleared bloodstream infections in experimental endocarditis in rabbit models caused by vancomycin-resistant $S$. aureus. Efficacy of treatment with vancomycin in combination with aminoglysides against MRSA has also been tested (Lee et al., 2003). In the study of Miranda-Nowales (2006), synergy was evident for dicloxacillin or cephalotin in combination with amikacin against methicillin-resistant Staphylococcus spp.

In this research it has been shown that there is a chance to obtain an efficient antibiotic therapy in combination also in infections caused by CNS. Combinations of vancomycin and cephalotin against $S$. epidermidis, staphylococcus most frequently isolated from gram-positive hospital infections, seem to be particularly promising. A synergistic effect on growth inhibition of multiresistant S. haemolyticus strains was obtained using oxacillin and vancomycin as well as cephalotin and rifampicin. However, clinical research including species identification of CNS causing infections is necessary, because as can be seen, individual species of this group differ in antibiotic resistance.

\section{Acknowledgements}

This study was supported by a grant 503-3012-3 from Medical University of Łódź.

\section{Literature}

Bourgeois I., M. Pestel-Caron, J. Lemeland, J.L. Pons and F. Caron. 2007. Tolerance to the glycopeptides vancomycin and teicoplanin in coagulase-negative staphylococci. Antimicrob. Agents Chemiother. 51: 740-743.

Chandran A.U. and R. Rennie. 2005. Routine antimicrobial susceptibility testing of coagulase-negative staphylococci isolated from blood cultures: is it necessary? Clin. Microbiol. Infect. 11: 1037-1040. Climo M.W, R.L. Patron and G.L. Archer. 1999. Combinations of vancomycin and $\beta$-lactams are synergistic against staphylococci with reduced susceptibilities to vancomycin. Antimicrob. Agents Chemiother. 43: 1747-1753.

Clinical and Laboratory Standards Institute. 2008. Performance standards for antimicrobial Susceptibility testing. Eighteenth Informational Supplement. M100-S18. CLSI, Wayne, PA, USA.

Dawis M.A., H.D.Isenberg, K.A. France and S.G. Jenkins. 2003. In vitro activity of gatifloxacin alone and in combination with cefepime, meropenem, piperacillin and gentamicin against multidrug-resistant organism. J. Antimicrob. Chemother. 51: 1203-1211. 
Finkelstein R., R. Fusman, I. Oren, I. Kassis and N. Hashman. 2002. Clinical and epidemiologic significance of coagulase-negative staphylococci bacteremia in a tertiary care university Israeli hospital. Am. J. Infect. Control. 30: 21-5

Fox P.M., R.J. Lampen, K.S. Stumpf, G.L. Archer and M.W. Climo. 2006. Succesful therapy of experimental endocarditis caused by vancomycin-resistant Staphylococcus aureus with a combination of vancomycin and $\beta$-lactam antibiotics. Antimicrob. Agents Chemiother. 50: 2951-2956.

Frigatto E.A., A.M. Machado, A.C. Pignatari and A.C. Gales. 2005. Is the cefoxitin disk test reliable enough to detect oxacillin resistance in coagulase-negative staphylococci? J. Clin. Microbiol. 43: 2028-2029.

Guerrero M.L.F. and M. Górgolas. 2006. Comparative activity of cloxacillin and vancomycin against methicillin-susceptible Staphylococcus aureus experimental endocarditis. J. Antimicrob. Chemother. 58: 1066-1069.

Hamels S., J.L. Gala, S. Dufour, P. Vannuffel, N. Zammatteo and J. Remacle. 2001. Consensus PCR and microarray for diagnosis of the genus Staphylococcus species, and methicillin resistance. Biotechniq. 31: 1364-6

John M.A., C. Pletch and Z. Hussain. 2002. In vitro activity of quinupristin/dalfopristin, linszolid, telithromycin and comparator antimicrobial agents against 13 species of coagulase-negative staphylococci. J. Antimicrob. Chemother. 50: 933-938.

Kloos W.E. 1997. Taxonomy and systematic of staphylococci indigenous to humans. pp. 123-125. In: Crossley K.B. and G.L. Archer (eds). The Staphylococci in Human Disease. Churchill Livingstone, London.

Kloos W.E. and T.L. Bannerman. 1994. Update on clinical significance of coagulase-negative staphylococci. Clin. Microbiol. Rev. 7: $117-140$.

Krediet T.G., M.E. Jones, L.J. Gerards and A. Fleer. 1999. Clinical outcome of cephalotin versus vancomycin therapy in the treatment of coagulase-negative staphylococcal septisemia in neonates: Relation to methicillin resistance and mec A gene carriage of blood isolates. Pediatrics 103: 3

Kuti J.L., C.R.V. Kiffer, C.M.F. Mendes and D.P. Nicolau. 2008. Pharmacodynamics comparsion of linezolid, teicoplanin and vancomycin against clinical isolates of Staphylococcus aureus and coagulase-negative staphylococci collected from hospitals in Brasil. Clin. Microbiol. Infect. 14: 116-123.

Lawrence S.L., V. Roth, R. Slinger, B. Toye, I. Gaboury and B. Lemyre. 2005. Cloxacillin versus vancomycin for presumed lateonset sepsis in the Neonatal Intensive care Unit and the impact upon outcome of coagulase negative staphylococcal bacteremia: a retrospective cohort study. BMC Pediatrics 5: 49.
Lee D., H. Chun, D. Yim, S.M. Choi, J.H. Choi, J.H. Yoo, W.S. Shin and M.W. Kang. 2003. Efficacies of vancomycin, arbekacin, and gentamicin alone or in combination against methicillin-resistant Staphylococcus aureus in an in vitro infective endocarditis model. Antimicrob. Agents Chemiother. 47: 3768-3773.

Martins A. and R.S. Cunha Maria de Lourdes. 2007. Methicillin resistance in Staphylococcus aureus and coagulase-negative staphylococci: Epidemiological and molecular aspects. Microbiol. Immunol. 51: 787-795.

Miranda-Nowales G., B.E. Leanos-Miranda, M. Vilchis-Perez and F. Solórzano-Santos. 2006. In vitro activity effects of combinations of cephalotin, dicloxacillin, imipenem, vancomycin and amikacin against methicillin-resistant Staphylococcus spp. strains. Ann. Clin. Microb. Antimicrob. 5: 25.

Nowak T, E. Balcerczak, M. Mirowski and E.M. Szewczyk. 2006. Detection of methicillin resistance in hospital environmental strains of coagulase-negative staphylococci. Pol. J. Microbiol. 55: 339-343. Szewczyk E.M., A. Piotrowski and M. Różalska. 2000. Predominant staphylococci in the intensive care unit of a pediatric hospital. J. Hosp. Infec. 45: 145-154.

Szewczyk E.M., M. Różalska, T. Cieślikowski and T. Nowak. 2004. Plasmids of Staphylococcus cohnii isolated from the intensive-care unit. Folia Microbiol. 49: 123-131.

Tacconelli E., E.M.C. D'Agata and A.W. Karchmer. 2003. Epidemiological comparison of true methicillin-resistant and methicillinsusceptible coagulase-negative staphylococcal bacteremia at hospital admission. Clin. Infect. Dis. 37: 644-649.

Tan T.Y., S.Y. Ng, W.X. Ng. 2006. Clinical significance of coagulase-negative Staphylococci recovered from nonsterile sites. J. Clin. Microbiol. 44: 3413-3414.

Van Griethuysen A., M. Pouw, van Leewen M. Heck, P. Willemse, A. Buiting, and J. Kluytmans. 1999. Rapid slide latex agglutination test for detection of methicillin resistance in Staphylococcus aureus. J. Clin. Microbiol. 37: 2789-2792.

Walsh T.R., A. Bolmström, A. Qwärnström, P. Ho, M. Wootton, R.A. Howe, A.P. MacGowan, and D. Diekema. 2001. Evaluation of current methods for detection of Staphylococci with reduced susceptibility to glycopeptides. J. Clin. Microbiol. 39: 2439-2444.

Wang Y.C. and M. Lipstich. 2006. Upgrading antibiotic use within a class: Tradeoff between resistance and treatment success. PNAS. 103: 9655-9660

Weinstein M.P., S. Mirrett, L. van Pelt, M. Mc-Kinnon, B.L. Zimmer, W. Kloos and L.B. Reller. 1998. Clinical importance of identifying coagulase-negative staphylococci isolated from blood cultures: Evaluation of microscan rapid and dried overnight gram-positive panels versus a conventional reference method. J. Clin. Microbiol. 36: 2089-2092 